\title{
A new meshless method using Taylor series to solve elasticity problems
}

\author{
Yendoubouam Tampango ${ }^{\mathrm{a}}$, Michel Potier-Ferry $^{\mathrm{a} *}$, Yao Koutsawa ${ }^{\mathrm{b}}$ and Salim Belouettar ${ }^{\mathrm{b}}$ \\ ${ }^{a}$ Laboratoire LEM3, UMR CNRS 7239, Ile du Saulcy, 57045 Metz cedex 01, France; ${ }^{b}$ CRP Henri Tudor \\ Luxembourg, 66 Rue de Luxembourg, L-4221 Esch sur Alzette, Luxembourg
}

\begin{abstract}
A meshless method is presented and analysed. In this approach, one discretises only the boundary, the partial differential equation being solved in the domain by using Taylor series expansion. A least square method is used to apply boundary conditions. In this paper, the method is applied to Navier equations for linear elasticity. Various tests are presented to discuss the efficiency and robustness of the method. The convergence is exponential with respect to the degree but it depends on the radius of convergence of the series. That is why an algorithm has been associated with the Domb-Sykes plot that is a classical method to detect singularities and evaluate the radius of convergence.

Dans ce travail nous présentons et analysons une nouvelle méthode sans maillage. Dans cette nouvelle méthode l'EDP est résolue de manière exacte dans le domaine en utilisant des séries de Taylor. Les conditions aux limites sont prises en compte par une technique des moindres carrés couplée à la technique de collocation. Cette méthode est appliquée à un problème d'élasticité linéaire. Plusieurs études de convergence ont été faites afin de s'assurer de l'efficacité de la méthode proposée. Ensuite en se basant sur le critère de Domb Sykes, une technique a été proposée pour estimer le rayon de convergence des séries solutions à partir des coefficients de Taylor calculés.
\end{abstract}

Keywords: PDE; meshless method; convergence analysis; Taylor series expansion; DombSykes plot

Mots-clés: EDP; méthodes sans maillages; analyse de convergence; Domb Sykes plot

\section{Introduction}

In the last decades, there have been many advances about meshless methods. They are considered as a promising alternative to overcome the difficulties due to mesh generation. Indeed, the meshless methods do not require any mesh grid generation. The meshless methods are usually divided into two main categories: the boundary type meshless methods and the domain type meshless methods. Recently, Zézé, Potier-Ferry, and Damil (2010) have proposed a new meshless method belonging to the boundary type meshless method, only boundary discretisation is needed. In this technique, the governing differential equation is satisfied exactly in the domain by using a Taylor series expansion. This leads to a smaller system, which is solved by applying boundary conditions. The boundary conditions are taken into account by using a least square method as proposed by Zhang, Liu, Song, and Lu (2001).

*Corresponding author. Email: michel.potierferry@univ-metz.fr

ISSN 1779-7179 print/ISSN 1958-5829 online

(C) 2012 Taylor \& Francis

http://dx.doi.org/10.1080/17797179.2012.721500

http://www.tandfonline.com 
This method has several advantages. First, the number of shape functions is much smaller than with the p-version of finite elements (Babuska, Szabo, \& Katz, 1981; Campion \& Jarvis, 1996). Second, the partial differential equation (PDE) is solved quasi-exactly inside the domain so that only the discretisation of the boundary is needed. Lastly, it can converge very rapidly with the degree ( $\mathrm{p}$-convergence).

In the Taylor series approximation, the domain of validity of the approximated solution is given by a circle centred at the development point of the series. The circle of convergence of the Taylor series is limited by the closest singularity to the domain. Hence, the development point must be chosen in a zone from which the total boundary must be visible. This can always be achieved by subdividing the total domain in subdomains. Hence, the following questions arise: how to choose the development point of the series? When the subdividing of the domain is needed? It is clear that the answer to these questions depends on the available information on the function to be approximated. Those informations are not directly accessible because the function to be approximated is the unknown solution of the PDE studied. Then, it becomes necessary to extract these informations from the calculated Taylor coefficients. Generally, the radius of convergence of a series is determined by the behaviour of its coefficients at infinity. But in the proposed method, one computes only a finite number of coefficients. However, in the literature, there exist several techniques to find the nearest singularity from a finite number of coefficients: Domb-Sykes plot, Darboux criterion, Pade approximant (Baker \& Graves-Morris, 1996; Domb \& Sykes, 1961; Garajeu, Cochelin, \& Medale, 2010; Hunter \& Guerrieri, 1980; Van Dyke, 1974).

In this paper, the proposed method is addressed and applied to a linear elastic problem. The second section presents a brief description of the technique. In Section 3, an application is made on the Navier equation of linear elasticity. Several tests of convergence have been made on two different types of solutions: a polynomial solution and a rational solution with a singularity. In the last section, the Domb-Sykes plot is applied on the series solution, which leads to a criterion which allows to estimate a possible singularity of a given problem from the calculated coefficients.

\section{Implementation of the technique}

In this section, a brief description of the technique is given. A detailed description can be seen in Zézé (2009) and Zézé et al. (2010). Beyond having the advantages of mesh-free methods, the method proposed here can handle more effectively the boundary conditions, which is usually a challenge for mesh-free methods. In order to build a high degree boundary meshless method, the main idea of the technique is to make a Taylor series approximation. Thus, in $2 \mathrm{D}$, one can write the unknown of a PDE in the form:

$$
u^{h}(x, y)=\sum_{k=0}^{N} \sum_{i=0}^{k} u_{i, k-i} x^{i} y^{k-i}=\sum_{k=0}^{N}\left\langle X^{k}\right\rangle\left\{u^{k}\right\}
$$

where

$$
\left\langle X^{k}\right\rangle=\left\langle x^{k}, x^{k-1} y, \ldots, y^{k}\right\rangle, \quad\left\langle u^{k}\right\rangle={ }^{t}\left\{u^{k}\right\}=\left\langle u_{k, 0}, u_{k-1,1}, \ldots, u_{0, k}\right\rangle .
$$

For each degree $k$, the unknown is the vector $\left\{u^{k}\right\} \in \mathbb{R}^{k+1}$. Then, for the complete polynom (1), there are $(N+1)(N+2) / 2$ coefficients to be found. The technique is split in two steps. 
In a first time, one makes a direct resolution of the PDE by using approximation (1). Then, the polynomial form (1) is introduced directly in the PDE and one makes an identification according to the order of the variables $\left\langle X^{k}\right\rangle$ for all $k, 0 \leq k \leq N-2$. This identification leads to a family of linear equations with fewer equations than unknowns. The resolution of these equations can then be made by determining a part of unknowns like a function of the other ones. Then, one can rewrite the approximate form (1):

$$
u^{h}(x, y)=\sum_{k=0}^{N}<P_{k}>\left\{v^{k}\right\}=\langle P(x, y)\rangle\{v\} .
$$

In (2), $\left\langle P_{k}>\right.$ are polynomial functions that are quasi-exact solutions of the PDE. The vectors $\left\{v^{k}\right\}$ are composed of coefficients remaining after resolution of the previous linear equations. These vectors are the new unknowns of the problem. Then, one reduces the number of unknows. For example, for a second order PDE, one goes from $(N+1)(N+2) / 2$ unknown coefficients in (1) to $2 N+1$ unknown coefficients in (2). The determination of these last coefficients will be the goal of the second step.

To achieve the resolution of the problem, one will apply the boundary conditions in order to find vectors $\left\{v^{k}\right\}$. In the literature, there are several techniques to take into account the boundary conditions. To reduce the computational cost, we prefer to use techniques avoiding numerical integration. At first, a technique of collocation has been used. It was shown in (Zézé, 2009) that this technique can become unstable. Then, the collocation technique has been replaced by a least square method as proposed by Zhang et al. (2001). One chooses a set of nodes on the boundary and one minimises the error between the approximate solution and the boundary data on these nodes. It comes to minimise the function:

$$
J(v)=\frac{1}{2} \sum_{j=1}^{M}\left|u^{h}\left(\underline{x}_{j}\right)-u_{j}^{d}\left(\underline{x}_{j}\right)\right|^{2} .
$$

This minimisation leads to a linear system $K\{v\}=F$ where $K$ is a symetric invertible matrix. Solving this system gives the vector $\{v\}$ and therefore the approximate solution of the problem.

Remark that various boundary conditions can be accounted in a similar way, see Tampango, Potier-Ferry, Koutsawa, and Belouettar (2012) where Neumann and Robin conditions were considered. Because the boundary conditions are satisfied in a mean square sense, they are not exactly verified in the same way as the PDE that is satisfied in the sense of Taylor series. The accuracy of these approximations will be discussed in Section 3.

\section{Validation of the technique}

\subsection{Problem}

Let us consider a linear elastic material in a plane domain $\Omega$ with boundary $\Gamma$, subjected to Neumann and Dirichlet boundary conditions. The relations governing the plane strain linear elasticity problem are given by Navier equations as follows:

$$
\left\{\begin{array}{l}
(\lambda+\mu) \operatorname{grad}(\operatorname{div}(\underline{u}))+\mu \Delta \underline{u}=0 \quad \text { in } \Omega \\
\text { Boundary conditions on } \Gamma
\end{array}\right.
$$

where $\lambda$ and $\mu$ are elastic constant and $\underline{u}$ is the displacement vector. 
From the Muskhelishvili's (1958) complex variable formalism for plane isotropic elastic problems, the solutions of this problem are given by:

$$
2 \mu \underline{u}=\kappa \phi(z)-z \overline{\phi^{\prime}(z)}-\overline{\psi(z)},
$$

where $\phi(z)$ and $\psi(z)$ are two holomorphic functions called complex potential functions of the complex variable $z=x+i y$.

In this paper, the considered domain is a disc with Dirichlet boundary conditions. In the numerical tests, we shall try to find analytical solutions in the form (5) with $\phi(z)=0$.

\subsection{Resolution of the PDE in the domain}

In the proposed method, one solves problem (4) by making the following polynomial approximation:

$$
\underline{u}^{h}(x, y)=\left\{\begin{array}{l}
u_{x}^{h}(x, y) \\
u_{y}^{h}(x, y)
\end{array}\right\}=\left\{\begin{array}{l}
\sum_{k=0}^{N}<X^{k}>\left\{u_{x}^{k}\right\} \\
\sum_{k=0}^{N}<X^{k}>\left\{u_{y}^{k}\right\}
\end{array}\right\}=\sum_{k=0}^{N}\left[X_{2}^{k}\right]\left\{u^{k}\right\}
$$

with

$$
\left\{u^{k}\right\}=\left\{\begin{array}{l}
\left\{u_{x}^{k}\right\} \\
\left\{u_{y}^{k}\right\}
\end{array}\right\} \quad \text { and } \quad\left[X_{2}^{k}\right]=\left[\begin{array}{cc}
<X^{k}> & 0 \\
0 & <X^{k}>
\end{array}\right]
$$

The unknowns are the vectors $\left\{u^{k}\right\}$. This approximate form is introduced in Equation (4) and an identification will be made related to the order of the monom $\left[X_{2}^{k}\right]$. For that, one must calculate the action of the differential operators appearing in (4) on the approximate form (6). In Zézé (2009), this action was already calculated and it has been shown that every mathematical operator is identified by matrices such that:

$$
L \underline{u}^{h}(x, y)=\sum_{k=0}^{N-l}\left[X_{2}^{k}\right]\left[L^{k}\right]\left\{u^{k+l}\right\}
$$

where $l$ is the order of the differential operator $L$. Let $\left[G^{k}\right],\left[D i v^{k}\right]$ and $\left[\Delta^{k}\right]$ being the matrices which define, respectively, the differential operators gradient, divergence and Laplacian (a detailed calculation of these matrices can be seen in Zézé (2009)). The problem (4) leads to:

$$
(\lambda+\mu)\left[G^{k}\right]\left[\operatorname{Div}^{k+1}\right]\left\{u^{k+2}\right\}+\mu\left[\Delta^{k}\right]\left\{u^{k+2}\right\}=0 \quad \forall k, \quad 0 \leq k \leq N-2
$$

For each $k$, this last relation is a family of $2 \times(k-1)$ equations for $2 \times(k+1)$ unknowns. Then, it permits to find a part of the vector $\left\{u^{k}\right\}$ as a function of the complementary components. This then leads to:

$$
\underline{u}^{h}(x, y)=\sum_{k=0}^{N}\left[P^{k}(x, y)\right]\left\{v^{k}\right\}
$$




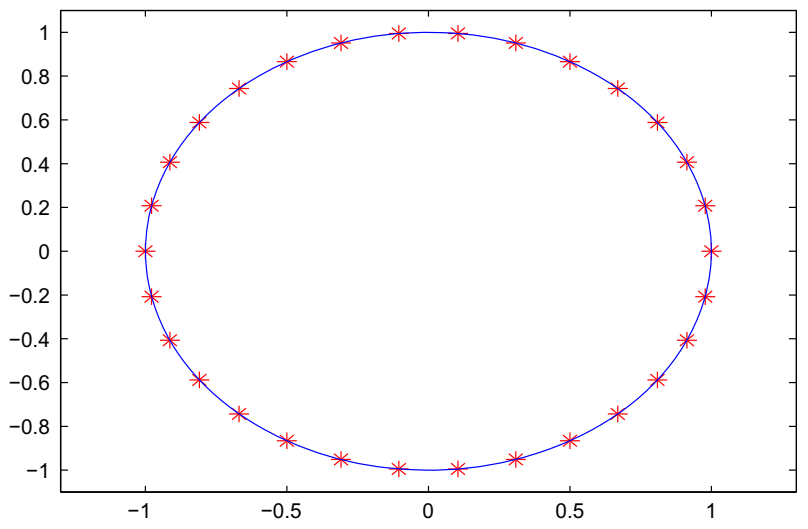

Figure 1. Discretisation of the domain for boundary conditions application.

The vectors $\left\{v^{k}\right\}$ are a part of vectors $\left\{u^{k}\right\}$ and will be found by applying boundary conditions. To apply the boundary conditions, one chooses a set of nodes on the boundary as shown in Figure 1 and then one verifies the relation (3) on these nodes in a least square sense as explained in Section 2.

\subsection{Results}

In this section, the comparison between the approximate solution and the exact solution is addressed and analysed. We will be interested by the $x$-component of the displacement $\underline{u}$. Then, the solution of the problem studied will be $2 \mu u_{x}=-\operatorname{Re}(\psi(z))$. We present the results for two types of solution: a polynomial form with $\psi(z)=z^{5}+z$ and a rational form $\psi(z)=1 /\left(z-z_{0}\right)$, with a singularity at $z_{0}$.

Figure 2 shows the maximum error on an inner circle of radius $r=.9$. One notes that the convergence depends on the nature of the analytical solution. Indeed, for the polynomial solution, the best convergence is reached for degree 5 (degree of the analytical solution). This confirms that with the Taylor series approximation one can get quasi-exactly polynoms. Figure 2, (right) presents the error for the rational solution for two points of singularity. It is clear that the position of the singularity influences the convergence. Indeed, for a point of singularity far from the domain, one has a very fast convergence. Thus, one reaches error $10^{-15}$ at order 30 . The convergence is worse if the singular point is close to the domain, nevertheless we have a sufficient convergence of $10^{-3}$ at order 20 . One can improve this error by increasing the degree of approximation. Therefore, a high degree approximation can be made.

Figure 3 presents the distribution of the error across the domain. The approximation is very good inside the domain. This is due to the exact resolution made in the domain. The error increases when approaching the boundary. This error is introduced by the application of boundary conditions. The figure also shows that the error is bigger in the direction of the singularity. The error in the application of boundary conditions is represented by the last loop of Figure 3 and in this case the error on boundary conditions is lower than $.5 \%$. It can be much smaller for large degrees, as shown by Figures 2 and 4.

Figure 4 presents the influence of the development point on the convergence for the rational problem with singularity at $z_{0}=1+i$. For small degree, a variation of the point of development does not affect strongly the convergence of the method. Then, the proposed 

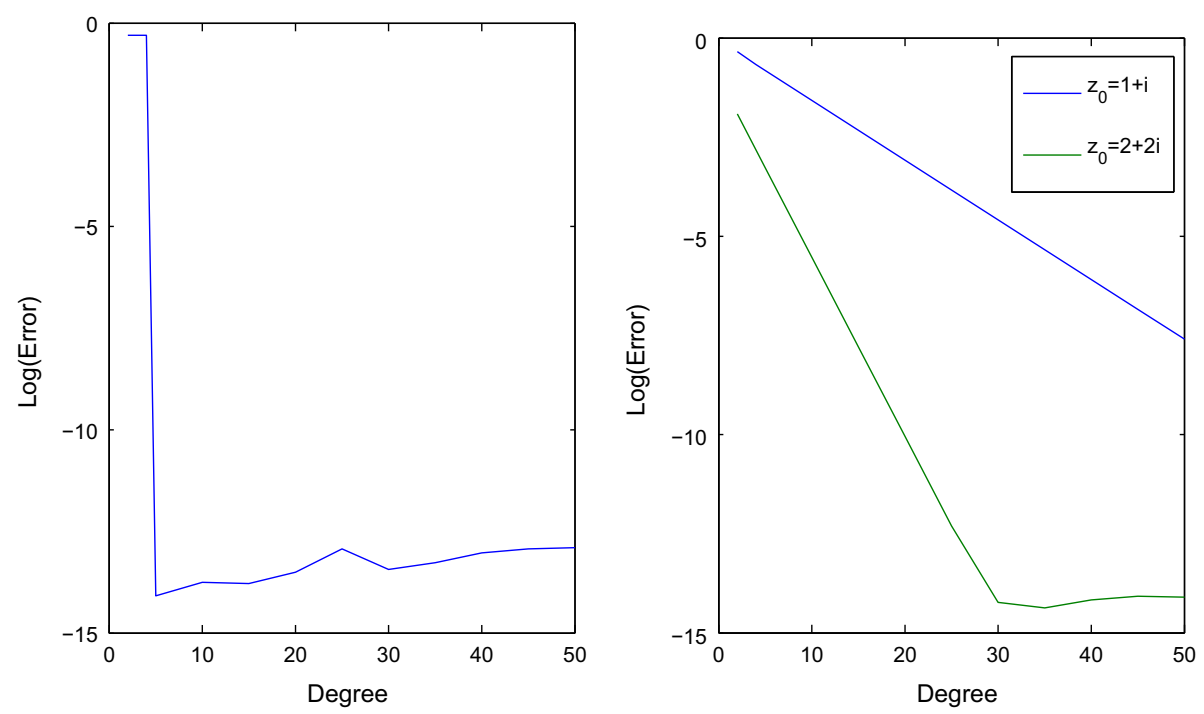

Figure 2. P-convergence. Left: polynomial solution $\psi(z)=z^{5}+z$. Right: rational solutions $\psi(z)=1 /\left(z-z_{0}\right)$.

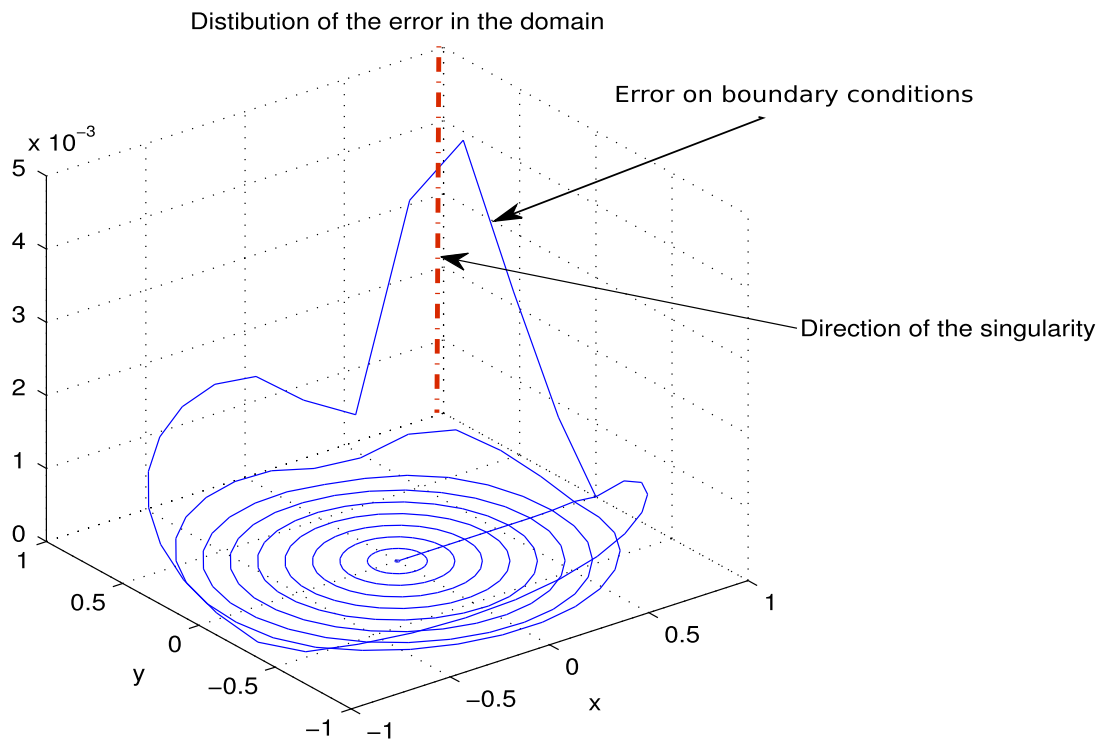

Figure 3. Distribution of the error across the domain.

method is stable regardless of the point of development. Therefore, when increasing the degree the convergence is not the same for any development point. The best convergence is obtained for the development point in the centre of the domain. This is due to the fact that the convergence domain of series is a circle. Therefore, one must choose the point of development in such a way that the entire domain be covered by the convergence domain. In the literature, the radius of convergence is often approached by the nearest singularly. This confirms the finding that the best choice of development point is the centre. 


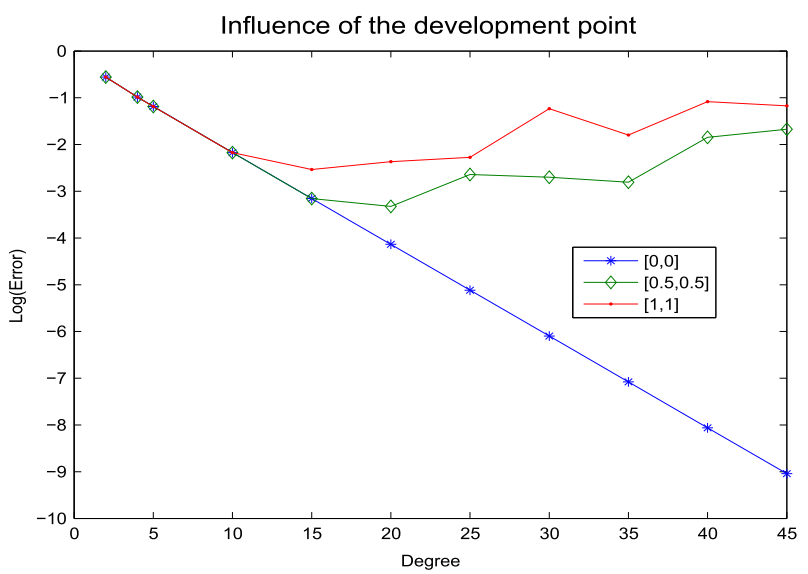

Figure 4. Influence of the development point.

\section{Convergence analysis}

For the problem studied here, the right choice for the point of development was expected because the geometry of the domain is simple and the analytical solution is known. On the contrary, for a problem with complex geometry which we do not know the analytical solution, it would be difficult to determine that point of development without a prior study. For some problems, we note that the proposed method cannot be applied to the whole domain and this will require to subdivide the domain in several subdomains. Generally, for series approximations, the radius of convergence is given by the nearest singularity. The purpose of this section is finding a technique of determination of the nearest singularity.

In approximation methods by series, the problem often encountered is related to the domain of convergence. The domain of validity of the approximation series is limited by the nearest singularity. Thus, in the resolution of PDE by series approximation, one must choose the development point in such a way that the domain of convergence covers the whole domain. However, the choice of the development point is not obvious. It is not always possible to have a development point in the domain such that the domain of validity covers the whole domain. In these cases, the domain is subdivided into several subdomains. Thus, in every subdomain, the development point can be chosen such that the domain of convergence covers the entire sub domains. This operation is only possible if one knows the position of the nearest singularity. So, the big problem is the determination of the nearest singularity without the knowledge of the analytical solution.

In the literature, there are several techniques to find the nearest singularity. Among others we can cite the Domb-Sykes plot, the criterion of Darboux, the Pade approximants, etc. All these methods analyse univariate functions, while the solution of the PDE is a function of two variables $u(x, y)$. These techniques will be applied on some lines of the plane, for instance lines parallel to $o x$ by considering the function $x \rightarrow u(x, y)$ or radial lines in the $\theta$ direction by considering $r \rightarrow u(r \cos \theta, r \sin \theta)$.

Here, one introduces only the Domb-Sykes plot. One obtains the same conclusions for the Darboux criterion, as established in Tampango et al. (2012).

The Domb-Sykes plot technique allows to estimate the nearest real singularity by looking at the behaviour of the series coefficients for large orders. The Domb-Sykes plot is prompted by the convergence criterion of D'Alembert. From this criterion, the radius of convergence of a series is the upper limit of the ratio $c_{n-1} / c_{n}$. This calculation seems impossible when one 
knows only a limited number of coefficients. Therefore, one must estimate this ratio. Domb and Sykes (1961) shows that the inverse ratio $c_{n} / c_{n-1}$ is often a linear function of $1 / n$ for $n$ sufficiently large.

Indeed, for a singular function of type (singularity at $\pm x_{0}$ ):

$$
f(x) \equiv \text { const } \times \begin{cases}\left(x_{0} \pm x\right)^{v}, & v \neq 0,1,2, \ldots \\ \left(x_{0} \pm x\right)^{v} \log \left(x_{0} \pm x\right), & v=0,1,2, \ldots\end{cases}
$$

the coefficients of its power series satisfy:

$$
D_{s}(i)=\frac{c_{i}}{c_{i-1}}= \pm \frac{1}{x_{0}}\left(1-\frac{1+v}{i}\right) .
$$

In Figure 5, the Domb-Sykes plot is presented along two radial lines $\theta=\pi / 4$ and $\theta=\arctan \left(y_{0} / x_{0}\right)$. The estimate (11) is not valid in the direction $\theta=\pi / 4$ and in most of the directions that we have considered because the ratio $c_{n} / c_{n-1}$ oscillates for large $n$. On the contrary, in the direction of the exact singularity, the ratio $c_{n} / c_{n-1}$ is nearly constant and this constant is exactly the inverse of $\left\|z_{0}\right\|$ that is the exact radius of convergence. This plot predicts an order $\alpha=-1$, which is consistent with the exact solution $\operatorname{Re}\left(1 /\left(z-z_{0}\right)\right)$.

The tests performed with the criterion of Darboux have given the same result. The criterion is only satisfied by considering the series in the direction of the singularity. In this direction, the ratio $D_{s}(N)$ converges to the inverse of the radius of convergence and the predicted order of singularity is about -1 .

A reliable criterion for finding the location of singularity will be to determine first the direction and subsequently its position. Hence, it seems that an efficient algorithm to detect the nearest singularity in the plane would be defined by analysing the ratio $D_{s}(N)$ corresponding to the Domb-Sykes plot in many radial lines. The direction of the singularity is the one such that the ratio $D_{s}(N)$ converges for large $N$ and it can be defined by a numerical criterion:

$$
\left\|D_{s}(N)-D_{s}(N-1)\right\| \leq \epsilon
$$

where $\epsilon$ is a chosen very small number.
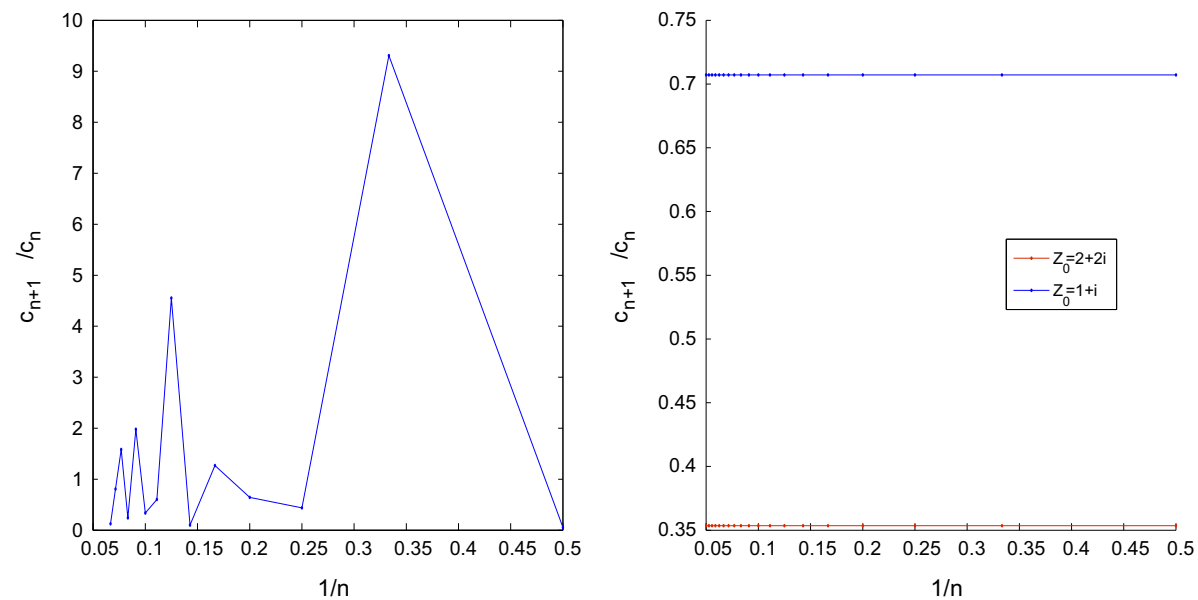

Figure 5. Domb-Sykes plot for the linear elasticity problem with rational solution. Left: direction $\pi / 4$. Right: direction of the singularity. 


\section{Conclusion}

In this paper, we have presented a new meshless method with application to a linear elastic problem. This method is characterised by the fact that the PDE is solved quasi-exactly in the domain, requiring only the discretisation of the boundary. The boundary conditions are taken into account by using a least square method hence no need for numerical integration. These aspects combine to give an efficient high-degree boundary meshless method. However, the convergence depends on the problem studied. The study of the linear elastic problem has shown a good convergence depending on degree and location of the nearest singularity. This is due to the fact that we are using Taylor series approximation. Consequently, in the second part of the paper, we proposed a criterion for a numerical determination of the radius of convergence from the Taylor coefficients implemented. The criterion proposed in this paper is based on the technique of the Domb-Sykes plot but other techniques exist that can lead to a similar result.

In this paper, we limited ourselves to compressible linear elasticity, but of course it can be extended to many differential equations. For instance, in Tampango (2009), a similar procedure has been used for the incompressible case and in Taro (2012), it has been applied to a second non-linear PDE. In the case of non-linear equations, a Newton algorithm is introduced to define a sequence of linearised equations that are further solved by the procedure described in the present paper. For an application to a more complex geometry, see Figure 6 of Zézé et al. (2010).

\section{References}

Babuska, I., Szabo, B.A., \& Katz, I.N. (1981). The p-version of the finite element method. SIAM Journal on Numerical Analysis, 18, 515-545.

Baker, G.A., \& Graves-Morris, P. (1996). Padé approximants. Cambridge: Cambridge University Press.

Campion, S.D., \& Jarvis, J.L. (1996). An investigation of the implementation of the p-version finite element method. Finite Elements in Analysis and Design, 23(1), 1-21.

Domb, C., \& Sykes, M.F. (1961). Use of series expansions for the Ising model susceptibility and excluded volume problem. Journal of Mathematical Physics, 2(1), 63-67.

Garajeu, D., Cochelin, B., \& Medale, M. (2010). Analyse et amélioration des séries (Analysis and improvement of series), Technical report, Laboratoire de Mécanique et d' Acoustique.

Hunter, C., \& Guerrieri, B. (1980). Deducing the properties of singularities of functions from their Taylor series coefficients. SIAM Journal on Applied Mathematics, 39(2), 248-263.

Muskhelishvili, M. (1958). Some basic problems of the mathematical theory of elasticity (pp. 113-115). Groningenp: Noordhoff.

Tampango, Y. (2009). Résolution des problèmes incompressibles de Stockes par la méthode de perturbation (Solving incompressible Stokes problems by a perturbation method) (Master's thesis, Université de Metz).

Tampango, Y., Potier-Ferry, M., Koutsawa, Y., \& Belouettar, S. (2012). Convergence analysis and detection of singularities within a boundary meshless method based on Taylor series. Engineering Analysis with Boundary Elements, 36(10), 1465-1472.

Taro, M. (2012). Elaboration d' un algorithme numérique permettant de résoudre un problème d'élasticité non linéaire à l' aide d' une méthode developpée au LEM3 (A numerical algorithm to solve a nonlinear elasticity problem by a method developed in LEM3)(Master's thesis, Université de Lorraine).

Van Dyke, M. (1974). Analysis and improvement of perturbation series. Quarterly Journal of Mechanics and Applied Mathematics, 27, 423-450.

Zézé, D. (2009). Calcul de fonctions de forme de haut degré par une technique de perturbation (Computating high degree shape functions by a perturbation technique) ( $\mathrm{PhD}$ thesis, Université Paul Verlaine Metz).

Zézé, D.S., Potier-Ferry, M., \& Damil, N. (2010). A boundary meshless method with shape functions computed from the PDE. Engineering Analysis with Boundary Elements, 34(8), 747-754.

Zhang, X., Liu, X.H., Song, K.Z., \& Lu, M.W. (2001). Least-squares collocation meshless method. International Journal for Numerical Methods in Engineering, 51(9), 1089-1100. 